This item was submitted to Loughborough's Institutional Repository (https://dspace.lboro.ac.uk/) by the author and is made available under the following Creative Commons Licence conditions.

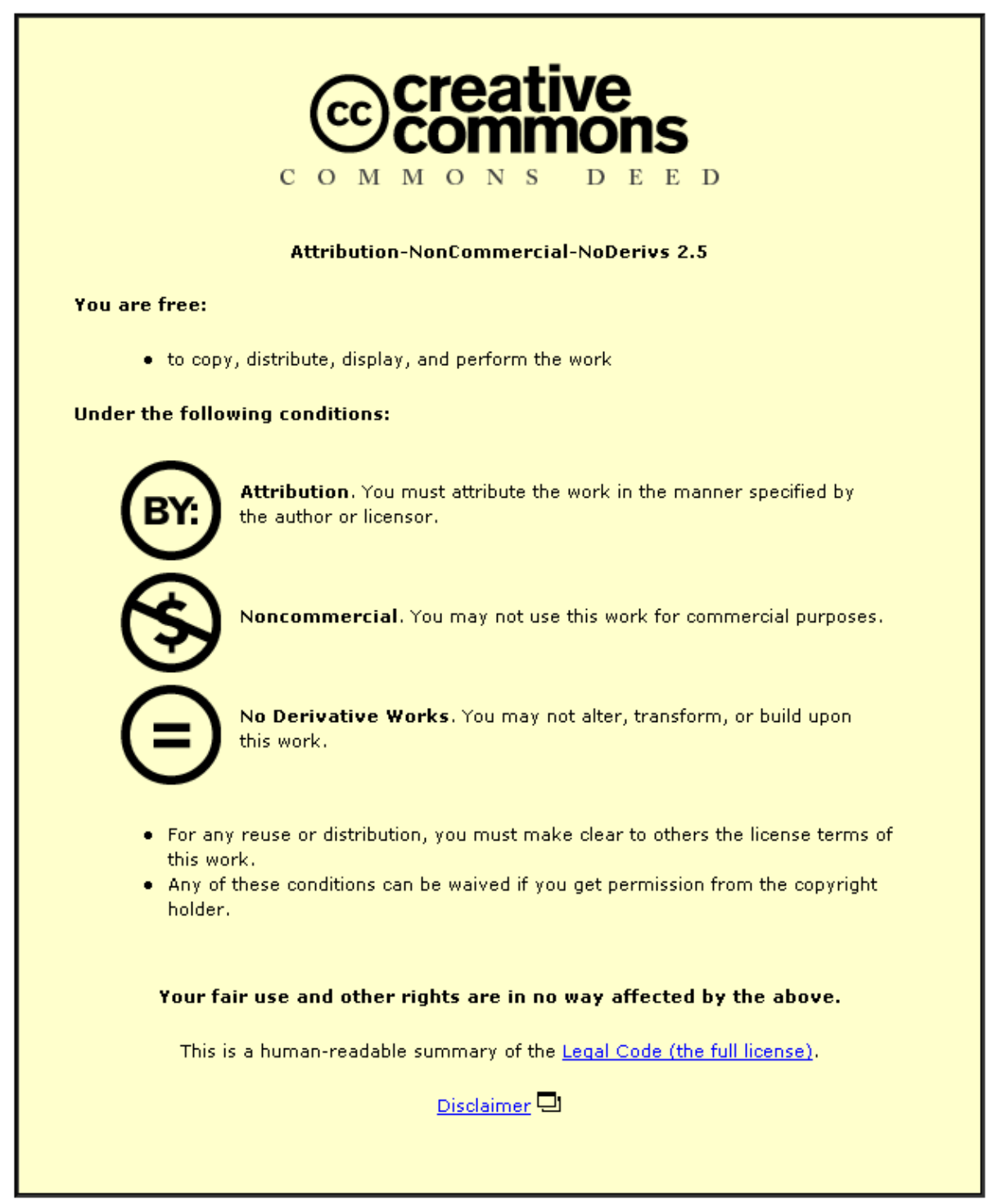

For the full text of this licence, please go to: http://creativecommons.org/licenses/by-nc-nd/2.5/ 


\title{
Low-Complexity Iterative Method of Equalization for Single Carrier With Cyclic Prefix in Doubly Selective Channels
}

\author{
Sajid Ahmed, Mathini Sellathurai, Sangarapillai Lambotharan, and Jonathon A. Chambers
}

\begin{abstract}
Orthogonal frequency division multiplexing (OFDM) requires an expensive linear amplifier at the transmitter due to its high peak-to-average power ratio (PAPR). Single carrier with cyclic prefix (SC-CP) is a closely related transmission scheme that possesses most of the benefits of OFDM but does not have the PAPR problem. Although in a multipath environment, SC-CP is very robust to frequency-selective fading, it is sensitive to the time-selective fading characteristics of the wireless channel that disturbs the orthogonality of the channel matrix (CM) and increases the computational complexity of the receiver. In this paper, we propose a time-domain low-complexity iterative algorithm to compensate for the effects of time selectivity of the channel that exploits the sparsity present in the channel convolution matrix. Simulation results show the superior performance of the proposed algorithm over the standard linear minimum mean-square error (L-MMSE) equalizer for SC-CP.
\end{abstract}

Index Terms-Computational complexity, doubly selective channel, iterative equalization.

\section{INTRODUCTION}

$\mathbf{I}$ $\mathrm{N}$ ORTHOGONAL frequency division multiplexing (OFDM), an inverse fast Fourier transform (IFFT) of a block of data symbols generally yields a time-domain signal that has very large peak-to-average power ratio (PAPR). The front-end power amplifier at the transmitter often is not linear in operation over the wide range of signal amplitudes. Therefore, unlike other modulation schemes, OFDM generally requires very expensive linear front-end power amplifiers. Single carrier with cyclic prefix (SC-CP) is a closely related transmission scheme, which possesses similar attractive multipath interference mitigation properties as OFDM and can exploit the FFT to design a computationally efficient frequency domain equalizer (FDE) [1]. In SC-CP, the symbols are transmitted directly without performing an IFFT operation; thereby, SC-CP avoids the PAPR problem and does not demand an expensive linear power amplifier [2], [3]. In order to design a low-complexity receiver for a SC-CP system, both the FFT and an IFFT operations are performed at the receiver. The baseband model for an SC-CP transmission scheme is shown in Fig. 1. Here,

Manuscript received March 17, 2005; revised June 23, 2005. The associate editor coordinating the review of this manuscript and approving it for publication was Dr. Hongbin Li.

S. Ahmed, M. Sellathurai, and J. A. Chambers are with the Centre of Digital Signal Processing, Cardiff University, Cardiff CF24 OYF, U.K. (e-mail: ahmeds6@cf.ac.uk; sellathuraiM@cf.ac.uk; chambersJ@cf.ac.uk).

S. Lambotharan is with the Centre of Digital Signal Processing Research, King's College London, London WC2R 2LS, U.K. (e-mail: s.lambotharan@ kcl.ac.uk).

Digital Object Identifier 10.1109/LSP.2005.860552

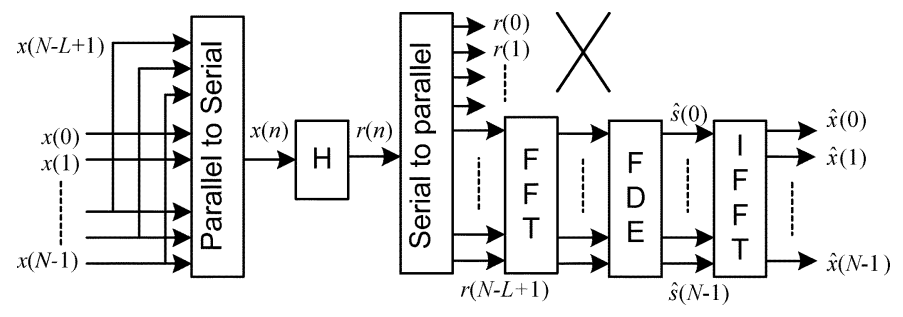

Fig. 1. Basic baseband SC-CP transmission and reception system, transmitting subsequent blocks of $N$ data symbols and performing frequency domain equalization at the receiver.

as in OFDM, a cyclic prefix is appended before transmission at the beginning of the time samples and removed at the receiver. In order to design the FDE, an FFT is performed on the received time samples to convert them into the frequency domain samples. Frequency domain equalization is then performed with the assumption that channel state information is available, and then the transmitted time-domain samples are obtained by an IFFT applied to the estimated frequency domain samples. Performing the FFT on the received time-domain samples yields an orthogonal CM that facilitates the design of a low-complexity equalizer. Frequency domain equalization for a SC-CP system applied to a frequency-selective channel is discussed in [4] and the references therein. When the channel is both frequency and time selective, it is termed doubly selective and may disturb the orthogonality of the $\mathrm{CM}$, which thereby degrades the performance of the FDE and renders the equalization computationally very expensive. In order to perform frequency domain equalization for an SC-CP in a doubly selective channel, Schniter et al. [5] preprocessed the time-domain received signal by multiplying with window coefficients that squeezed the significant coefficients in the CM into $2 D+1$ central diagonals, where $D=f_{d} N+1$, in which $f_{d}$ and $N$ are, respectively, the Doppler shift (DS) and the number of symbols in the SC-CP block. The complexity of this algorithm depends on the DS. In contrast to this paper, if we examine the time-domain model of the received OFDM signal, the channel convolution matrix is already sparse and has similar structure to that after preprocessing of the received samples [5], [6]. The number of nonzero elements in a row depends on the length of the channel taps $L$, which for a wireless channel is typically of the order of four [7]. In environments with more scattering, channel shortening algorithms can be used to shorten the channel length [8]. Sparsity of the channel convolution matrix can be exploited to design a low-complexity SC-CP equalizer for doubly selective channels. Therefore, working with time 
samples, an efficient low-complexity iterative algorithm is proposed that does not need preprocessing, and the complexity is independent of the DS.

The letter is organized as follows. In the following section, the signal model is presented. Then in Section III, we study symbol estimation based on an MMSE equalizer and iterative updating. In Section IV, we discuss the complexity of the algorithm. Simulation results are given in Section V, followed by our conclusions in Section VI.

Notations: Bold upper case $\mathbf{X}$ denotes a matrix, and lower case $\mathbf{x}$ a vector. The $n$th row and $l$ th column entry of a matrix $\mathbf{X}$ is denoted by $\mathbf{X}_{n, l}$. Transposition and conjugate transposition of a matrix are, respectively, denoted by $(.)^{T}$ and $(.)^{H}$; $\operatorname{diag}(\mathbf{x})$ is a diagonal matrix with diagonal elements taken from the vector $\mathbf{x} . \mathbf{I}_{N}$ is an identity matrix of size $N . \operatorname{Pr}\{\},. p\{$.$\} ,$ $E\{\},. \operatorname{Re}\{$.$\} , and \langle.\rangle_{N}$ denote, respectively, the probability of the discrete event in the bracket, probability of the continuous event, statistical expectation, the real part of a complex number, and the modulo- $N$ operation. Finally, we use $\{x(n)\}$ to denote the sequence $x(n)$ of $N$ symbols, where $n=0,1, \cdots, N-1$ and $\operatorname{Cov}[x(n), y(n)]$ to represent the covariance between $x(n)$ and $y(n)$.

\section{PROBlem STATEMENT}

The basic baseband model for an SC-CP system is given in Fig. 1. Let us suppose that the number of symbols in an SC-CP block is $N$ and the signal has propagated through $L$ different paths. Considering that the sampling rate is equal to the symbol transmission rate, the received baseband signal sampled at discrete time $n$ after removing the cyclic prefix can be written as

$$
\begin{aligned}
r(n) & =\sum_{l=0}^{L-1} h_{n, l} x\left(\langle n-l\rangle_{N}\right)+v(n) \\
& =\frac{1}{\sqrt{N}} \sum_{k=0}^{N-1} s(k) e^{j \frac{2 \pi}{N} k n} \sum_{l=0}^{L-1} h_{n, l} e^{-j \frac{2 \pi}{N} k l}+v(n)
\end{aligned}
$$

where $h_{n, l}$ is the channel gain (CG) for the $l$ th channel tap, $x(n)$, $v(n)$, and $s(k)$ are, respectively, the transmitted time-domain signal, the zero mean circularly complex white Gaussian noise at sample time $n$, and the frequency domain transmitted signal at frequency $k$. The estimation of time-variant and time-invariant channels is, respectively, discussed in [9] and [10]. Throughout this paper, we assume perfect knowledge of CGs. The $N$ received samples in vector form can be written as

$$
\mathbf{r}=\mathbf{H} \mathbf{x}+\mathbf{v}=\mathbf{H F}^{H} \mathbf{s}+\mathbf{v} .
$$

Taking the FFT of the received signal yields

$$
\mathbf{F r}=\mathbf{F H F}^{H} \mathbf{S}+\mathbf{F v}=\mathbf{H}_{d f} \mathbf{s}+\mathbf{F v}
$$

where $\mathbf{H}$ is the time-domain channel convolution matrix of size $N \times N$, the $n$th row and $l$ th column entry of the matrix $\mathbf{H}$ can be written as $\mathbf{H}_{n, l}=h_{n,\langle n-l\rangle_{N}}$. Moreover, $\mathbf{F}$ is the FFT matrix of size $N \times N, \mathbf{H}_{d f}$ is the $\mathrm{CM}$, and $\mathbf{s}$ is a vector of frequency domain transmitted signals. The MMSE equalizer for the estimation of $\mathbf{s}$ can be found by minimizing $E\left\{\|\mathbf{s}-\mathbf{W F r}\|^{2}\right\}$, yielding

$$
\mathbf{W}=\left(\mathbf{H}_{d f}^{H} \mathbf{H}_{d f}+\sigma_{n}^{2} \mathbf{I}_{N}\right)^{-1} \mathbf{H}_{d f}^{H}
$$

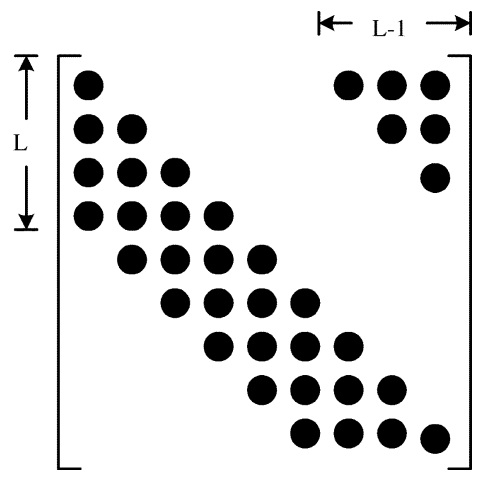

Fig. 2. Diagonal structure of the time-domain channel convolution matrix $\mathbf{H}$, where $L$ is the channel support.

where $\sigma_{n}^{2}$ is the variance of the noise. For frequency domain equalization, if in (3) the channel is linear time invariant (LTI), then the matrix $\mathbf{H}_{d f}$ will be diagonal. Thereby, in order to estimate the sequence of samples $\{s(k)\}$, the L-MMSE equalizer requires the inversion of a diagonal matrix that is computationally inexpensive. Furthermore, to estimate the sequence of transmitted symbols $\{x(n)\}$, an inverse FFT is performed on the sequence of estimated samples $\{s(k)\}$.

On the other hand, in a linear time-variant (LTV) channel, the matrix $\mathbf{H}$ is not circulant; therefore, the matrix $\mathbf{H}_{d f}$ is not diagonal, a consequence of which is that intercarrier interference (ICI) is introduced. Hence, the L-MMSE equalizer requires the inversion of an $N \times N$ Hermitian matrix that needs $\mathcal{O}\left(N^{2}\right)$ operations, which is infeasible for large $N$ and yields poor bit-error rate (BER) performance [9]. However, as shown in Fig. 2, if modulo- $N$ indexing is assumed, then the structure of $\mathbf{H}$ reveals that the individual symbol $x(n)$ contributes only to the observation samples $r(n)$ to $r(n+L-1)$. Therefore, considering modulo- $N$ indexing in the sequel, it can be noted that these are the only samples required to estimate $x(n)$, and in vector form, these received samples can be written as $\mathbf{r}_{n}=\mathbf{H}_{n} \mathbf{x}+\mathbf{v}_{n}$, where $\mathbf{r}_{n}=\left[\begin{array}{llll}r(n) & r(n+1) & \cdots & r(n+L-1)\end{array}\right]^{T}$, matrix $\mathbf{H}_{n}$ contains $L$ rows of the matrix $\mathbf{H}$ from $n$ to $n+L-1$ and $\mathbf{v}_{n}=\left[\begin{array}{lllll}v(n) & v(n+1) & \cdots & v(n+L-1)\end{array}\right]^{T}$.

\section{SYMbOL ESTIMATION}

By exploiting the sparsity present in the time-domain channel convolution matrix, the transmitted symbols $\{x(n)\}$ can be estimated without performing explicit FFT and inverse FFT operations. On this basis, we next describe a low-complexity MMSEiterative algorithm.

\section{A. MMSE Equalizer}

The noise is assumed uncorrelated and zero mean; therefore, $E\left\{\mathbf{v}_{n}\right\}=\mathbf{0}, E\left\{\mathbf{v}_{n} \mathbf{v}_{n}^{H}\right\}=\sigma_{n}^{2} \mathbf{I}_{L}$, and $E\left\{x(n) \mathbf{v}_{n}\right\}=\mathbf{0}$. Moreover, we define $\bar{x}(n)=E\{x(n)\}, \overline{\mathbf{x}}=E\{\mathbf{x}\}, c_{x}(n)=$ $\operatorname{Cov}[x(n), x(n)]$, and $\mathbf{c}_{x}=\left[\begin{array}{llll}c_{x}(0) & c_{x}(1) & \cdots & \left.c_{x}(N-1)\right] \text {. The }\end{array}\right.$ MMSE equalizer $\mathbf{w}_{n}$ of length $L$ for the soft estimates of $x(n)$ is given by [5], [6]

$$
\begin{aligned}
& \mathbf{w}_{n}=\left(\mathbf{H}_{n} \operatorname{diag}\left(\mathbf{c}_{x}\right) \mathbf{H}_{n}^{H}+\sigma_{x}^{2} \mathbf{I}_{L}\right)^{-1} \mathbf{h}_{n} c_{x}(n) \\
& \text { and the estimate } \hat{x}(n)=\bar{x}(n)+\mathbf{w}_{n}^{H}\left(\mathbf{r}_{n}-\mathbf{H}_{n} \overline{\mathbf{x}}\right)
\end{aligned}
$$


with the assumption that $\{\bar{x}(n) \neq 0\}$, the mean values of the estimates of the individual symbols cannot be equal to zero, and in (5), $\mathbf{h}_{n}$ is the $n$th column of $\mathbf{H}_{n}$.

\section{B. Iterative Algorithm}

We wish to find the a posteriori values of $\{\bar{x}(n)\}$ and $\left\{c_{x}(n)\right\}$ to use in (5) and (6). To find these values, the following steps are required to form the proposed iterative algorithm.

Step 1) In the first iteration, we initialize all the mean values $\{\bar{x}(n)\}=0$ that corresponds to $\operatorname{diag}\left(\mathbf{c}_{x}\right)=\mathbf{I}_{N}$; then, the estimate $\hat{x}(n)$ is obtained using (5) and (6).

Step 2) With the assumption of binary phase shift keying (BPSK) signals, it is more convenient to work with the log-likelihood ratios (LLRs) rather than the probabilities [12]. The $a$ priori and a posteriori LLR of $x(n)$ are defined as [13]

and

$$
L[x(n)]=\ln \left(\frac{\operatorname{Pr}\{x(n)=1\}}{\operatorname{Pr}\{x(n)=-1\}}\right)
$$

$$
L\left[\left.x(n)\right|_{\hat{x}(n)}\right]=\ln \left(\frac{\operatorname{Pr}\left\{x(n)=\left.1\right|_{\hat{x}(n)}\right\}}{\operatorname{Pr}\left\{x(n)=-\left.1\right|_{\hat{x}(n)}\right\}}\right) .
$$

The difference between the a posteriori and a priori LLRs (which is the extrinsic information) of $x(n)$ is

$$
\begin{aligned}
\triangle L[x(n)] & =L\left[\left.x(n)\right|_{\hat{x}(n)}\right]-L[x(n)] \\
& =L\left[\left.\hat{x}(n)\right|_{x(n)}\right] \\
& =\ln \frac{p\left\{\left.\hat{x}(n)\right|_{x(n)=1}\right\}}{p\left\{\left.\hat{x}(n)\right|_{x(n)=-1}\right\}} .
\end{aligned}
$$

In order to find the extrinsic LLR, $L\left[\left.\hat{x}(n)\right|_{x(n)}\right]$, it is assumed that the probability density function (PDF) of $\hat{x}(n)$ is Gaussian with variance $\sigma_{x}^{2}$ and can be written as $p\{\hat{x}(n)\}=\left(1 / \sqrt{2 \pi} \sigma_{x}\right) \exp (-((\hat{x}(n)-E\{\hat{x}(n)\})(\hat{x}(n)-$ $\left.\left.E\{\hat{x}(n)\})^{H}\right) / 2 \sigma_{x}^{2}\right)$. Therefore, the conditional PDF, when the transmitted signal $x(n)=b \epsilon\{+1,-1\}$, of $\hat{x}(n)$ becomes $p\left\{\left.\hat{x}(n)\right|_{x(n)=b}\right\}=\left(1 / \sqrt{2 \pi} \sigma_{x}\right) \exp \left(-\left(\left(\hat{x}(n)-m_{n}(b)\right)(\hat{x}(n)-\right.\right.$ $\left.\left.\left.m_{n}(b)\right)^{H}\right) /\left.2 \sigma_{x}^{2}\right|_{x(n)=b}\right)$, where $m_{n}(b)=E\left\{\left.\hat{x}(n)\right|_{x(n)=b}\right\}$ and $\left.\sigma_{x}^{2}\right|_{x(n)=b}=\operatorname{Cov}\left[\hat{x}(n),\left.\hat{x}(n)\right|_{x(n)=b}\right]$, which are the conditional mean and variance of $\hat{x}(n)$.

Throughout the iterative receiver process, we exchange only extrinsic information. That is, when estimating $x(n)$, we use only the a priori information from $\{x(k), k \neq n\}$. Therefore, it is assumed that the a priori information $\bar{x}(n)=0$ and $c_{x}(n)=$ 1 in (5) and (6). Hence, the conditional mean can be determined by using (6) as

$$
E\left\{\left.\hat{x}(n)\right|_{x(n)=b}\right\}=\mathbf{w}_{n}^{H} \mathbf{h}_{n} b .
$$

It should be noted that $m_{n}(b)$ depends on the particular value of $b$. Similarly, it can be shown that the conditional variance of $\hat{x}(n)$ becomes

$$
\begin{aligned}
\left.\sigma_{x}^{2}\right|_{x(n)=b} & =E\left\{\left.\hat{x}(n) \hat{x}^{H}(n)\right|_{x(n)=b}\right\}-m_{n}(b) m_{n}(b)^{H} \\
& =E\left\{\left.\hat{x}(n) \hat{x}^{H}(n)\right|_{x(n)=b}\right\}-\mathbf{w}_{n}^{H} \mathbf{h}_{n} \mathbf{h}_{n}^{H} \mathbf{w}_{n}
\end{aligned}
$$

within which the term

$$
\begin{aligned}
& E\left\{\left.\hat{x}(n) \hat{x}^{H}(n)\right|_{x(n)=b}\right\} \\
& =\left.\mathbf{w}_{n}^{H}\left(\mathbf{H}_{n} \operatorname{diag}\left(\mathbf{c}_{x}\right) \mathbf{H}_{n}^{H}+\sigma_{n}^{2} \mathbf{I}_{L}\right) \mathbf{w}_{n}\right|_{x(n)=b} .
\end{aligned}
$$

Substituting (5) in (10) yields $E\left\{\left.\hat{x}(n) \hat{x}^{H}(n)\right|_{x(n)=b}\right\}=$ $\mathbf{w}_{n}^{H} \mathbf{h}_{n}$. Therefore, (9) becomes $\left.\sigma_{x}^{2}\right|_{x(n)=b}=\mathbf{w}_{n}^{H} \mathbf{h}_{n}-$
$\mathbf{w}_{n}^{H} \mathbf{h}_{n} \mathbf{h}_{n}^{H} \mathbf{w}_{n}$. Note that unlike the mean, the variance of the estimator is independent of $b$. Now, the difference between the a posteriori and the a priori LLR of $x(n)$ becomes

$$
\begin{aligned}
\triangle L[x(n)] & =\ln \frac{\left[\exp \left(-\frac{\left(\hat{x}(n)-m_{n}(+1)\right)^{2}}{\left.\sigma_{x}^{2}\right|_{x(n)=+1}}\right)\right]}{\left[\exp \left(-\frac{\left(\hat{x}(n)-m_{n}(-1)\right)^{2}}{\left.\sigma_{x}^{2}\right|_{x(n)=-1}}\right)\right]} \\
& =4 \frac{\operatorname{Re}\{\hat{x}(n)\}}{1-\mathbf{h}_{n}^{H} \mathbf{w}_{n}}
\end{aligned}
$$

and the $a$ posteriori LLR of $x(n)$

$$
L\left[\left.x(n)\right|_{\hat{x}(n)}\right]=L[x(n)]+\triangle L[x(n)] .
$$

Step 3) Exploiting (12) and using the property $\operatorname{Pr}\{x(n)=$ $\left.\left.1\right|_{\hat{x}(n)}\right\}+\operatorname{Pr}\left\{x(n)=-\left.1\right|_{\hat{x}(n)}\right\}=1$, the updated values for $\bar{x}(n)$ and $c_{x}(n)$ are obtained as

$$
\begin{aligned}
\bar{x}(n)_{\text {new }} & =\operatorname{Pr}\left\{x(n)=+\left.1\right|_{\hat{x}(n)\}}-\operatorname{Pr}\left\{x(n)=-\left.1\right|_{\hat{x}(n)\}}\right.\right. \\
& =\tanh \left(\frac{L\left[\left.x(n)\right|_{\hat{x}(n)}\right]}{2}\right) \\
\text { and } c_{x}(n)_{\text {new }} & =\sum_{b \in\{+1,-1\}}\left(b-\bar{x}(n)_{\text {new }}\right)^{2} \operatorname{Pr}\left(x(n)=\left.b\right|_{\hat{x}(n)}\right) \\
& =1-\bar{x}(n)_{\text {new }}^{2} .
\end{aligned}
$$

Note that (13) and (14) update the values of $\bar{x}(n)$ and $c_{x}(n)$ in (5) and (6) in Step 1).

Step 4) We repeat steps 1) through 3) until the specified number of iterations has elapsed.

\section{COMPLEXITY OF THE AlgORITHM}

\section{A. Linear Time-Variant Channel}

Although the dimensions of matrix $\mathbf{H}_{n}$ are $L \times N$, it contains only $2 L-1$ nonzero columns. In each iteration, to find the equalizer coefficient values $\mathbf{w}_{n}$, the algorithm requires the inversion of $\left[\mathbf{H}_{n} \operatorname{diag}\left(\mathbf{c}_{x}\right) \mathbf{H}_{n}^{H}+\sigma_{n}^{2} \mathbf{I}_{L}\right]$ that needs $\mathcal{O}\left(L^{2}\right)$ operations and must be performed $N$ times per iteration. The product term inside the matrix $\mathbf{H}_{n} \operatorname{diag}\left(\mathbf{c}_{x}\right) \mathbf{H}_{n}^{H}$ can be computed in $\mathcal{O}\left(L^{2}\right)$ operations as a consequence of the sparsity within the channel convolution matrix. Once $\mathbf{w}_{n}$ is obtained, the estimation of $\hat{x}(n)$ requires the computation of $\mathbf{H}_{n} \overline{\mathbf{x}}$ that also needs $\mathcal{O}\left(L^{2}\right)$ operations and must be repeated $N$ times per iteration. Therefore, to estimate $N$ symbols, we only require $\mathcal{O}\left(N L^{2}\right)$ operations per iteration.

\section{B. Linear Time-Invariant Channel}

In an LTI channel, the FDE requires $\mathcal{O}\left(2 N \log _{2} N\right)$ operations, while the proposed algorithm requires $\mathcal{O}\left(L^{2} N\right)$ operations per iteration. Therefore, the complexity of the proposed algorithm for a small length of channel $(\leq 5)$ is identical to the complexity of the FDE, but the performance is better.

\section{Simulation}

In this section, we compare the performance of our low-complexity MMSE-iterative algorithm after five iterations with the L-MMSE equalizer and match filter bound (MFB). The MFB is obtained from the model given in (2) by assuming the symbols $\left\{\left.x(l)\right|_{l \neq n}\right\}$ are known. The number of subcarriers is chosen to be $N=64$, and the length of the cyclic prefix is kept equal 


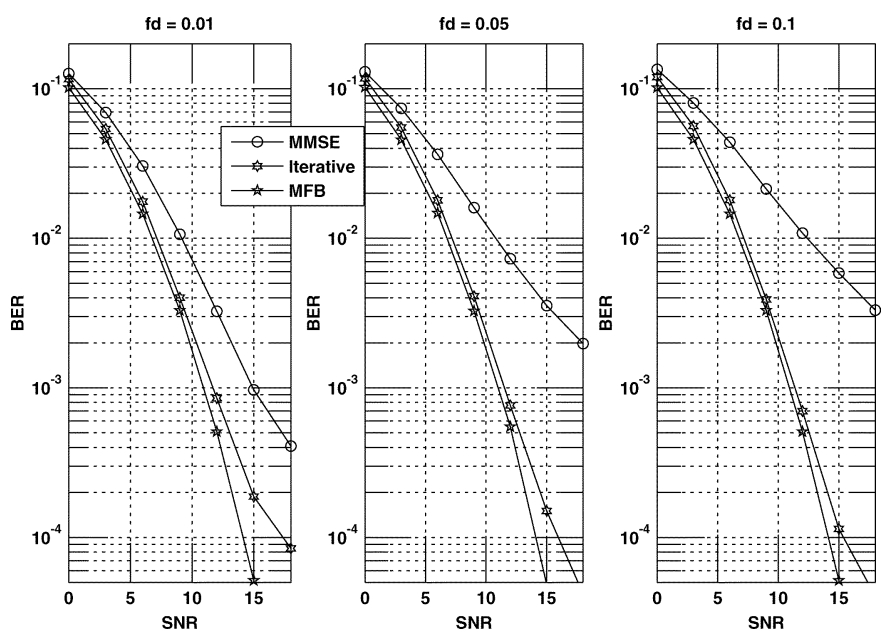

Fig. 3. BER performance comparison of the proposed iterative algorithm after five iterations with the L-MMSE equalizer and MFB at different DSs.

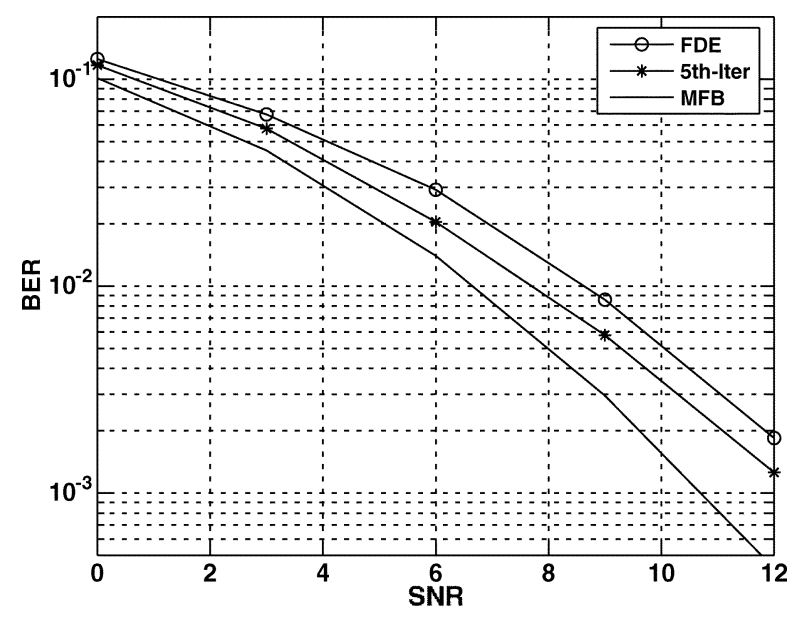

Fig. 4. BER performance comparison of the proposed iterative algorithm for an LTI channel after five iterations with the FDE and MFB.

to the length of the channel. We use a four-tap wireless fading channel model in which each channel tap is represented by a complex Gaussian random process independently generated with the Doppler spectrum based on Jakes' model. Here, we assume $\sum_{l=0}^{L-1} \sigma_{l}^{2}=1$, where $\sigma_{l}^{2}$ is the variance of the $l$ th path. The transmitted symbols $\{x(n)\}$ are BPSK. In order to examine the benefits of employing the proposed iterative method, BER performance is compared with the L-MMSE equalizer and MFB in Fig. 3. Low DS changes the channel very slowly, and in this case, the performances of the iterative method and the L-MMSE equalizer are close to each other. However, as the large DS introduces significant time selectivity into the channel, the proposed algorithm outperforms the L-MMSE equalizer. Importantly, our method exploits time diversity gain and thereby has performance very close to the MFB. In Fig. 4, the performance of the proposed algorithm is compared to that of the FDE for the case of an LTI channel. Even in the case of an LTI channel, the iterative method outperforms the FDE by at least $1 \mathrm{~dB}$.

\section{CONCLUSION}

We considered the design of a low-complexity iterative receiver for single and doubly selective channels. The simulation results demonstrate the superiority of the proposed scheme over the L-MMSE equalizer, which is not only computationally expensive but also has poor performance. On the other hand, unlike the iterative method proposed in [5], the computational complexity of our proposed algorithm is independent of frequency offsets, does not require preprocessing, and can work for a large range of frequency offsets without increasing the computational complexity significantly. The computational complexity of the proposed algorithm depends on the length of the channel that can be reduced by applying channel shortening algorithms.

\section{ACKNOWLEDGMENT}

The authors would like to thank Dr. P. Schniter for sharing his MATLAB code related to the work presented in [6]. They also would like to thank the anonymous reviewers for improving the clarity of the presentation of this letter.

\section{REFERENCES}

[1] H. Sari, G. Karam, and I. Jeanclaude, "Transmission techniques for digital terrestrial TV broadcasting," IEEE Commun. Mag., vol. 33, no. 2, pp. 100-109, Feb. 1995.

[2] G. L. Stuber, J. R. Barry, S. W. Mclaughlin, and M. A. Ingram, "Broadband MIMO-OFDM wireless communications," Proc. IEEE, vol. 92, no. 2, pp. 271-294, Feb. 2004.

[3] L. Deneire, B. Gyselinckx, and M. Engels, "Training sequence versus cyclic prefix-a new look on single carrier communication," IEEE Commun. Lett., vol. 5, no. 7, pp. 292-294, Jul. 2001.

[4] D. Falconer, S. L. Ariyavisitakul, A. B. Seeyar, and B. Edison, "Frequency domain equalization for single-carrier broadband wireless systems," IEEE Commun. Mag., vol. 6, no. 4, pp. 58-66, Apr. 2002.

[5] P. Schniter and H. Liu, "Iterative equalization for single-carrier cyclicprefix in doubly-dispersive channels," in Proc. Asilomar Conf. Signals, Systems, Computers, vol. 1, Nov. 2003, pp. 502-506.

[6] P. Schniter, "Low-complexity equalization of OFDM in doubly selective channels," IEEE. Trans. Signal Process., vol. 52, no. 4, pp. 1002-1010, Apr. 2004.

[7] Multiplexing and Multiple Access on Radio Path, April 2003. (3GPP TS 45.002 V5.9.0).

[8] J. Zhang, W. Ser, and J. S. Zhu, "Effective optimization method for channel shortening in OFDM systems," Proc. Inst. Elect. Eng. Commun., vol. 150, no. 2, pp. 85-90, Apr. 2003.

[9] Y. S. Choi, P. J. Voltz, and F. A. Cassara, "On channel estimation and detection for multi-carrier signals in fast and selective Rayleigh fading channels," IEEE Trans. Commun., vol. 49, no. 8, pp. 1375-1387, Aug. 2001.

[10] S. Ahmed, S. Lambotharan, A. Jakobsson, and J. A. Chambers, "Parameter estimation and equalization techniques for communication channels with multipath and multiple frequency offsets," IEEE Trans. Commun., vol. 53, no. 2, pp. 219-223, Feb. 2005.

[11] T. Kailath, Linear Estimation. Upper Saddle River, NJ: Prentice-Hall, 2000.

[12] R. Koetter, A. C. Singer, and M. Tuchler, "Turbo equalization," IEEE Signal Process. Mag., vol. 21, no. 1, pp. 67-80, Jan. 2004.

[13] M. Tüchler, R. Koetter, and A. C. Singer, "Turbo equalization: principles and new results," IEEE Trans. Commun., vol. 50, no. 5, pp. 754-766, May 2002. 